\title{
Haematological changes in Schistosoma haematobium infections in school children in Gabon
}

\author{
Jean Claude Dejon-Agobé ${ }^{1,2} \cdot$ Ayôla A. Adegnika ${ }^{1,3,4,5,6} \cdot$ Martin P. Grobusch ${ }^{1,2,4,7,8}$
}

Received: 23 September 2020 / Accepted: 30 December 2020 / Published online: 24 January 2021

(c) The Author(s) 2021

\begin{abstract}
Background Schistosomiasis is a parasitic disease affecting the blood cell. As a chronic disease, schistosomiasis particularly impacts on the human host's haematological profile. We assessed here the impact of urogenital schistosomiasis on the full blood counts (FBC) as proxy diagnostic tool for schistosomiasis.

Methods A cross-sectional study was conducted among school children living in Lambaréné, Gabon. Schistosomiasis status was determined using urine filtration technique. EDTA blood samples were analysed using a Pentra ABX $60^{\circledR}$ analyzer.

Results Compared to their infection-free counterparts, school children infected with Schistosoma haematobium displayed an altered FBC profile, with changes in all three blood cell lines. Adjusted for praziquantel intake, soil-transmitted helminthic infections and Plasmodium falciparum infection status, schistosomiasis was independently associated with a decreasing trend of mean haemoglobin $(\beta=-0.20 \mathrm{~g} / \mathrm{dL}, p$-value $=0.08)$ and hematocrit $(\beta=-0.61 \%, p$-value $=0.06)$ levels, a lower mean MCV $\left(\beta=-1.50 \mu \mathrm{m}^{3}, p\right.$-value $\left.=0.02\right)$ and $\mathrm{MCH}(\beta=-0.54 \mathrm{pg}, p$-value $=0.04)$, and higher platelet $\left(\beta=28.210^{3} / \mathrm{mm}^{3}\right.$, $p$-value $=0.002)$ and leukocyte $\left(\beta=1.1310^{3} / \mathrm{mm}^{3}, p\right.$-value $\left.=0.0003\right)$ counts, respectively.

Conclusions Schistosomiasis is associated with a characteristic FBC profile of schoolchildren living in Lambaréné, indicating the necessity to consider schistosomiasis as a single cause of disease, or a co-morbidity, when interpreting FBC in endemic areas.
\end{abstract}

Keywords Schistosomiasis $\cdot$ Schistosoma haematobium $\cdot$ Haematology $\cdot$ School children $\cdot$ Gabon

\section{Introduction}

Schistosomiasis is one of the two most-common parasitic infections globally, with transmission reported from 78 countries [1]. Globally, 700 million people live in endemic areas [2]. Among the now 240 million people estimated of

Martin P. Grobusch

m.p.grobusch@amsterdamumc.nl

1 Centre de Recherches Médicales de Lambaréné (CERMEL), Lambaréné, BP 242, Lambaréné, Gabon

2 Center of Tropical Medicine and Travel Medicine, Department of Infectious Diseases, Division of Internal Medicine, Amsterdam University Medical Centers, Location AMC, Amsterdam Public Health, Amsterdam Infection and Immunity, University of Amsterdam, Amsterdam, The Netherlands

3 Department of Parasitology, Leiden University Medical Center, 2333 ZA Leiden, The Netherlands having schistosomiasis and requiring treatment, more than $90 \%$ are estimated to live in sub-Saharan Africa [2, 3].

Schistosomiasis leads both to acute and chronic disease. At both stages, schistosomiasis stimulates the host immune system, being in part reflected in routinely measurable biochemical and, in focus here, haematological alterations. In general, higher mean leukocyte counts and changes in

4 Institut Für Tropenmedizin, Eberhard Karls University Tübingen, 72074 Tübingen, Germany

5 German Center for Infection Research, Partner Site Tübingen, 72074 Tübingen, Germany

6 Fondation Pour la Recherche Scientifique, 72 BP 045 Cotonou, Benin

7 Masanga Medical Research Unit (MMRU), Masanga, Sierra Leone

8 Institute of Infectious Diseases and Molecular Medicine (IIDMM), Cape Town, Republic of South Africa 
mean of differential leukocyte counts are observed among individuals infected with schistosomiasis compared to those without; both normalise following praziquantel (PZQ) treatment [4]. Eosinophilia is characteristic for schistosomiasis [5]. Thrombocytes play a protective role against schistosomiasis by exerting direct damaging effects on adult worms, with platelet count changes depending on the disease phase [6]. In mice, thrombocytes were shown to adhere to the surfaces of, and to kill mechanically transformed schistosomula, leading to thrombocytopenia in early disease [7]. In the brown rat, a three-fourfold increase of thrombocytes protective against schistosomes was observed 4-6 weeks after initial infection [8]. Few studies were conducted to assess thrombocyte changes in humans $[9,10]$, and the potential anthelminthic effects thereof. One study reported a thrombocyte reduction by half in patients with intestinal schistosomiasis. However, the authors concluded that this was more likely attributable to portal hypertension rather than directly to the helminths [9]. Another study reported a trend for thrombophilia among Ghanaian children with urogenital schistosomiasis compared to their non-infected counterparts [10]. In addition to leucocytes and platelets, the third blood cell line does not remain unaffected by schistosomiasis either. The main symptom of schistosomiasis is the presence of blood in urine (in urogenital schistosomiasis) [11] or in stool (in intestinal schistosomiasis), due to blood vessel rupture during egg excretion. Chronic schistosomiasis, which particularly affects school-aged children, adolescents and young adults has been reported to be associated with chronic inflammation and iron deficiency anaemia [12].

With schistosomiasis being mainly a chronic infection and very often asymptomatic at that stage, and knowing that schistosomiasis is able to influence the mean FBC parameters as discussed above, we can, thus, assume that in case of co-infections with other diseases such as malaria, schistosomiasis might eventually go unnoticed if the clinician is unaware of the presence of this (co)morbidity. Indeed, in malaria-negative individuals in co-endemic areas, for example, a certain level of microcytic anaemia on its own would probably go unnoticed as it is explained by repeated malaria episodes, with anaemia being one of the malariadiagnostic criteria for disease severity, and chronic anaemia being a well-recognised consequence of repeated malaria episodes. A recent epidemiological assessment demonstrated that Lambaréné is an area of urogenital schistosomiasis with a $26 \%$ prevalence, with hematuria and proteinuria being positively associated with the disease [13]. In this follow-up analysis, we examined the effect of schistosomiasis on FBCs as a surrogate diagnostic parameter, among school children living in Lambaréné, an area endemic for S. haematobium and where PZQ is available.

\section{Materials and methods}

\section{Study area}

Lambaréné is a town in Gabon located $80 \mathrm{~km}$ south of the equator known to be endemic for schistosomiasis. The predominant Schistosoma species is S. haematobium [13-16]. Lambaréné is also known to be endemic for soil-transmitted helminths (STH) [17] and malaria, with highest prevalences in school children and adolescents [18].

\section{Study design and population}

The study design was cross-sectional. Volunteers were recruited amongst consenting eligible, apparently healthy school children living in Lambaréné.

\section{Sample size consideration}

The current sample size was calculated for a cross-sectional study aiming to determine the prevalence of schistosomiasis in Lambaréné and associated factors, as described elsewhere [13]. In the current analysis, we assessed the difference in haematological parameters between participants with schistosomiasis and those without. Considering a 5\% type-I error, and having 161 participants included in the Schistosomapositive group and 451 in the control group, we were able, with more than $90 \%$ power, to detect a minimum of $10 \%$ between both groups for platelet levels, and more than $10 \%$ for WBC and RBC levels.

\section{Study procedures and laboratory examinations}

The study was conducted from April to July 2016. Participants were selected at school as described elsewhere [13]. Briefly, legal representatives of volunteers invited at school to partake were visited at home and asked to grant informed consent. Trained field workers used a standardised questionnaire to inquire with parents or other primary caretakers about, among other, history of passing blood in urine and treatment received (PZQ or other anthelminthic drugs) in the previous six months. Nurses collected study subjects' demographic data (age, sex and address) at school. In cases of acute medical concerns, the participant was referred to the clinician for appropriate care.

Eligible participants were provided with plastic containers at school and were invited to provide three urine samples on three consecutive days, and one stool sample at earliest convenience. For each urine sample, urine filtration was performed for the detection of S. haematobium eggs using a Whatman microfilter membrane of $10-12 \mu \mathrm{m}$ as described 
elsewhere [19]. We used the Kato-Katz technique for the detection of eggs of STH in stool samples as described elsewhere [20] and the copro-culture technique for the detection of hookworm (Necator americanus) larvae [13, 20]. One $2.7 \mathrm{~mL}$ blood sample was collected into an ethylenediaminetetraacetic acid (EDTA) tube to perform a FBC, using the automated haematology analyser (ABX Pentra $60^{\circledR}$, Horiba Instruments Incorp., Irvine, CA $<$ USA). In addition, $10 \mu \mathrm{L}$ of fingertip blood was collected for a thick blood smear (TBS) for the detection of Plasmodium spp. parasites applying the Lambaréné method [21]. A rapid diagnostic test (RDT) was performed for the rapid detection of circulating $P$. falciparum HRP-2 antigen using the Paracheck $P f^{\circledR}$ Malaria Test (Orchid Biomedical Systems, Goa, India).

Participants found positive for Schistosoma eggs were treated with $40 \mathrm{mg} / \mathrm{kg}$ of PZQ once. Those testing STHor Plasmodium positive were treated with oral albendazole $400 \mathrm{mg}$ daily for three consecutive days [22], or with artemisinin-based combination therapy (ACT) [23], respectively. For other infections, medical prescription was given by the study clinician, or the participant was referred to an appropriate health centre.

\section{Statistical considerations}

We collected data using the patient report form and digitalised it, using the REDCap electronic data capture tool [24] hosted at CERMEL. The original clean database was exported into R software (Version 3.2.4) and a subset database (see Supplementary Database) was obtained for the statistical analyses. Age was used as categorical variable, grouped in 5-year strata. Participants were considered having malaria when testing positive either with TBS or RDT. Qualitative variables were summarised by proportion and 95\% confidence interval (CI); while, quantitative variables were summarised by mean and standard deviation (SD) or by median and interquartile (IQ) range where appropriate. The normality of the distribution of continuous variables was assessed by visual inspection and if needed, a log transformation was performed. Chi-square test was used to compare qualitative variables. Student's $t$-test was used to compare means of continuous variables; while, the Wilcoxon test was used to compare their distribution. Linear Model (LM) regression was used to correct for confounding factors potentially influencing haematological parameters with regard to Schistosoma status. The residuals were used to check for assumptions to ensure the usefulness of the model. The level of statistical significance was set at less than 0.05 .

\section{Ethical considerations}

The original study protocol was approved by the institutional ethic committee of CERMEL (CEI-CERMEL 002/2016).
The study was conducted in line with the Good Clinical Practice (GCP) principles of the International Conference on Harmonization (ICH) [25] and the Declaration of Helsinki [26].

\section{Results}

\section{Study population characteristics}

A total of 614 participants were included in the original study [13], and 612 of them with haematology data available were incorporated in this analysis. The mean age of our study population was $10.1(\mathrm{SD}=2.7)$ years, with a 0.95 female:male ratio. The mean haemoglobin level was $11.6(\mathrm{SD}=1.13) \mathrm{g} / \mathrm{dL}$. Ten per cent $\left[{ }_{95 \%} \mathrm{CI}: 8-13\right]$ and $23 \%$ [95\% CI: 19-26] of our study population, respectively, declared having taken PZQ or albendazole treatment within the past six months prior to study enrolment. The prevalence of Plasmodium spp. infection combining TBS and RDT was $20 \%$ [95\% CI: $17-23$ ]. The prevalence of any STH was $15 \%$ [95\% CI: 12-18].

\section{Study group characteristics}

The 161 (26\%) participants found schistosoma-egg positive (159 in urine and two in faeces) constituted the schistosoma positive $(\mathrm{Sch}+)$ group; while their negative counterparts constituted the schistosoma-negative (Sch-) group, leading to a ratio of 2.8 non-infected participants per single infected participant (Table 1). The two groups were comparable for age $(p$-value $=0.33)$ and sex $(p$-value $=0.39)$. Of note, the proportion of the participants previously treated with PZQ was significantly higher among the $\mathrm{Sch}+$ group compared to the Sch- group $(16 \%$ vs $8 \%, p$-value $=0.008)$. The overall prevalence of STH was higher among the Sch + group compared to the Sch- group ( $22 \%$ vs $12 \%, p$-value $=0.005)$. Trichuriasis was the most prevalent STH infection in both groups, and was mostly prevalent among the Sch + group compared to the Sch- group (17\% vs $8 \%, p$-value $=0.001)$. Plasmodium infection was more prevalent in $\mathrm{Sch}+$ group than Sch- group ( $28 \%$ vs $17 \%$, $p$-value $=0.003)$, particularly when detected using RDT.

\section{Haematological profile and schistosomiasis status}

We assessed the haematological profile of the study participants according to schistosomiasis status (Table 2) in the absence of clinical signs or symptoms hinting at a possible concomitant infectious or non-infectious disease. We found significant lower haemoglobin levels among the schistosoma-positive participants compared to their schistosoma-negative counterparts (median: $11.5 \mathrm{~g} / \mathrm{dL}$ 
Table 1 Characteristics of the 612 study participants with regard to schistosomiasis infection status

\begin{tabular}{|c|c|c|c|c|c|c|c|c|c|}
\hline & & & \multicolumn{2}{|c|}{ Overall study population } & \multicolumn{2}{|c|}{$\begin{array}{l}\text { Schistosoma } \\
\text { negative }\end{array}$} & \multicolumn{2}{|c|}{$\begin{array}{l}\text { Schistosoma } \\
\text { positive }\end{array}$} & \multirow[t]{2}{*}{$p$ value } \\
\hline & & & $N$ & $\%, 95 \% \mathrm{CI}$ & $N$ & $(\%)$ & $n$ & $(\%)$ & \\
\hline \multicolumn{3}{|l|}{ Sample size } & 612 & - & 451 & (73.7) & 161 & $(26.3)$ & \\
\hline \multirow[t]{3}{*}{ Sex } & & & & & & & & & 0.39 \\
\hline & Female & & 299 & $48.9[40.2-48.2]$ & 225 & $(49.9)$ & 74 & $(46.0)$ & \\
\hline & Male & & 313 & $51.1[40.7-55.2]$ & 226 & $(50.1)$ & 87 & $(54.0)$ & \\
\hline \multicolumn{3}{|l|}{ Sex ratio (Female/Male) } & 0.95 & & 1.00 & & 0.85 & & \\
\hline \multicolumn{3}{|l|}{ Age (mean, sd) } & 10.1 & 2.7 & 10.1 & 2.7 & 10.0 & 2.8 & 0.83 \\
\hline \multirow[t]{4}{*}{ Age } & & & & & & & & & 0.33 \\
\hline & $5-9$ & & 352 & $57.5[53.5-61.5]$ & 262 & $(58.1)$ & 90 & $(55.9)$ & \\
\hline & $10-14$ & & 239 & $39.1[35.2-43.0]$ & 171 & $(37.9)$ & 68 & $(42.5)$ & \\
\hline & $15-19$ & & 21 & $3.4[2.1-5.2]$ & 18 & $(4.0)$ & 3 & (1.9) & \\
\hline \multicolumn{10}{|l|}{ Plasmodium infection* } \\
\hline & By microscopy & & 36 & $5.9[4.2-8.1]$ & 23 & $(5.1)$ & 13 & (8.2) & 0.16 \\
\hline & & Parasitemia (Geomet-ric mean) & & 756 & & 574 & & 1229 & 0.28 \\
\hline & By RDT & & 118 & $19.3[16.3-22.7]$ & 75 & $(16.7)$ & 43 & $(26.7)$ & 0.006 \\
\hline & Any method & & 122 & $20.1[17.0-23.5]$ & 77 & $(17.2)$ & 45 & $(28.3)$ & 0.003 \\
\hline \multicolumn{3}{|c|}{ History of praziquantel treatment** } & & & & & & & 0.008 \\
\hline & Yes & & 62 & $10.1[7.9-12.8]$ & 37 & (8.3) & 25 & $(15.7)$ & \\
\hline \multicolumn{10}{|l|}{$\mathrm{STH}^{* * *}$} \\
\hline & Ascariasis & & 15 & $3.2[1.8-5.2]$ & 7 & $(2.1)$ & 8 & $(6.1)$ & 0.03 \\
\hline & Trichuriasis & & 49 & $10.4[7.8-13.5]$ & 26 & (7.6) & 23 & $(17.4)$ & 0.001 \\
\hline & Hookworm & & 18 & $3.8[2.3-6.0]$ & 12 & $(3.5)$ & 6 & $(4.5)$ & 0.60 \\
\hline & Any STH & & 69 & $14.6[11.6-18.1]$ & 40 & $(11.8)$ & 29 & $(22.0)$ & 0.005 \\
\hline \multicolumn{3}{|c|}{ History of STH treatment $* * * *$} & & & & & & & 0.12 \\
\hline & Yes & & 137 & $22.6[19.3-26.1]$ & 108 & $(24.2)$ & 29 & $(18.1)$ & \\
\hline
\end{tabular}

$\$ 1$ missing data

*5 missing data; 4 for microscopy examination, 2 for rapid diagnosis test including 1 for both

**Taken in the last 6 months, 7 missing data

***140 missing data; 111 for Schistosoma-negative group and 29 for Schistosoma-positive group

$* * * * 5$ missing data

Table 2 FBC profile of the 612 study participants regarding schistosomiasis status

\begin{tabular}{lllll}
\hline FBC parameters & & \multicolumn{2}{l}{ Schistosoma status (median, [IQ]) } & $p$ value \\
\cline { 3 - 4 } & & Negative & Positive & (Wilcoxon test) \\
\hline Erythrocytes $\left(10^{6} / \mathrm{mm}^{3}\right)$ & & $\mathbf{4 . 5 6}[\mathbf{4 . 2 9 - 4 . 8 3}]$ & $\mathbf{4 . 5 4 , [ 4 . 3 0 - 4 . 8 5}]$ & $\mathbf{0 . 8 8}$ \\
& Haemoglobin $(\mathrm{g} / \mathrm{dl})$ & $11.7[11.1-12.3]$ & $11.5,[10.7-12.1]$ & 0.001 \\
& Hematocrit $(\%)$ & $35.9[34.2-35.8]$ & $35.4,[32.7-36.6]$ & 0.0005 \\
& MCV $\left(\mu \mathrm{m}^{3}\right)$ & $79.0[75.0-83.0]$ & $77.0,[73.0-81.0]$ & $<0.0001$ \\
& MCH $(\mathrm{pg})$ & $25.9[24.4-27.5]$ & $25.1,[23.7-26.5]$ & $<0.0001$ \\
Thrombocytes $\left(10^{3} / \mathrm{mm}^{3}\right)$ & & $32.7[32.0-33.2]$ & $32.5,[31.8-33.2]$ & 0.26 \\
Leukocytes $\left(10^{3} / \mathrm{mm}^{3}\right)$ & & $\mathbf{2 3 2}[\mathbf{1 7 0 - 2 8 7}]$ & $\mathbf{2 5 4 ,}[\mathbf{1 9 5}-\mathbf{3 1 5}]$ & $\mathbf{0 . 0 0 3}$ \\
& & $\mathbf{6 . 5 0}[\mathbf{5 . 5 0 - 8 . 0 0}]$ & $\mathbf{7 . 5 ,}[\mathbf{6 . 3 0 - 9 . 1 0}]$ & $<\mathbf{0 . 0 0 0 1}$ \\
& Lymphocytes $\left(10^{3} / \mathrm{mm}^{3}\right)$ & $3.24[2.64-4.40]$ & $3.57,[3.11-4.40]$ & $<0.0001$ \\
& Neutrophils $\left(10^{3} / \mathrm{mm}^{3}\right)$ & $2.29[1.79-3.09]$ & $2.49,[2.04-3.19]$ & 0.01 \\
& Eosinophils $\left(10^{3} / \mathrm{mm}^{3}\right)$ & $0.30[0.16-0.50]$ & $0.52,[0.33-0.90]$ & $<0.0001$ \\
& Basophils $\left(10^{3} / \mathrm{mm}^{3}\right)$ & $0.05[0.04-0.07]$ & $0.07,[0.05-0.09]$ & $<0.0001$ \\
& Monocytes $\left(10^{3} / \mathrm{mm}^{3}\right)$ & $0.39[0.03-0.61]$ & $0.43,[0.01-0.61]$ & 0.50 \\
\hline
\end{tabular}


vs $11.7 \mathrm{~g} / \mathrm{dL}, p$-value $=0.001)$. However, WBC $\left(7.510^{3} /\right.$ $\mathrm{mm}^{3}$ vs $6.510^{3} / \mathrm{mm}^{3}, p$-value $\left.<0.001\right)$ and thrombocyte $\left(25410^{3} / \mathrm{mm}^{3}\right.$ vs $23210^{3} / \mathrm{mm}^{3}$, p-value $\left.=0.002\right)$ counts were significantly higher in children with schistosomiasis. Looking specifically at the differential leukocyte count, we found a higher level of lymphocytes $\left(3.5710^{3} / \mathrm{mm}^{3}\right.$ vs 3.24 $10^{3} / \mathrm{mm}^{3}, p$-value $\left.<0.0001\right)$, neutrophils $\left(2.4910^{3} / \mathrm{mm}^{3}\right.$ vs $2.2910^{3} / \mathrm{mm}^{3}, p$-value $\left.=0.01\right)$, eosinophils $\left(0.5210^{3} / \mathrm{mm}^{3}\right.$ vs $0.3010^{3} / \mathrm{mm}^{3}$, $p$-value $\left.<10^{-4}\right)$ and basophils $\left(0.0710^{3} /\right.$ $\mathrm{mm}^{3}$ vs $0.0510^{3} / \mathrm{mm}^{3}$, p-value $\left.<0.001\right)$ in Sch + compared to Sch-.

A multivariate analysis was carried out to investigate a relationship between haematological constants and schistosomiasis status adjusted for PZQ intake, STH and P. falciparum infection status (Table 3 ), yielding a statistically significant positive correlation between thrombophilia and a diagnosis of schistosomiasis. Similarly, a statistically significant positive correlation between leukocyte count and a diagnosis of schistosomiasis was observed. This applied to all WBC subclasses except for monocytes, too (Table 3). For RBC, a relationship trend was found towards lower haemoglobin and haematocrit levels, respectively (Table 3). With regard to the corpuscular constants of RBCs, a significant relationship was observed for MCV ( $p$-value $=0.02)$ and MCH $(p$-value $=0.04)$, not for MCHC ( $p$-value $=0.83)$. Indeed, a decrease of 1.50 $\mu \mathrm{m}^{3}$ and $0.54 \mathrm{pg}$ was observed in MCV and $\mathrm{MCH}$ mean level of participants with schistosomiasis, respectively. The adjusted $\mathrm{R}^{2}$ value for each haematological constant was basically less than 0.01 ; so, less than $10 \%$ of the variation in these constants can be explained by our regression model. The data met the assumptions of homogeneity of variance and linearity and the residuals were approximately normally distributed.

\section{Discussion}

The main objective of the present analysis was to assess the effect of urogenital schistosomiasis on FBC parameters among schoolchildren living in Lambaréné, a semi-urban area. Our results reveal that children with schistosomiasis display an altered FBC profile with a change in the cell level count of the three cell types, as compared to those without the disease. However, taking into account some confounding factors which can either affect the haemoglobin and platelet levels [27] or schistosomiasis status such as PZQ treatment which is also known to normalise the leucocytes level in schistosomiasis infected children [4], the relationship between FBC parameters and schistosomiasis status observed was ambiguous for RBC; while, the observed change for thrombocyte and leukocyte counts was independently associated with the disease and was characterised by an increase in cell numbers.

Indeed, our results reveal a significant increase in total leukocyte count levels, as well as for each leukocyte type among children with schistosomiasis, as compared to those uninfected. The high WBC count levels we found among children with schistosomiasis, corroborate the reports of Mohammed et al. [4] and Afrifa et al. [10] among Sudanese and Ghanaian school children, respectively. We hypothesise that the high level of total leukocytes count observed is to be seen in connection with the host immune response against the presence of adult schistosomes in the bloodstream, and the particular role of eosinophils in the defense against helminthic infections is generally well recognised [28, 29]. Similarly, our results show a significant increase in thrombocyte count levels among $S$. haematobium-infected children. Based on what was demonstrated in brown rats [8], we hypothesise that thrombophilia is
Table 3 Differences in mean of the FBC parameters with regard to schistosomiasis status and adjusted on Plasmodium infection, any STH infection status, and history of praziquantel intake among the 472 participants with completed results

\begin{tabular}{lllll}
\hline FBC parameters & & \multicolumn{2}{l}{ Schistosoma positive } & \\
\cline { 3 - 4 } & & $\beta$ & {$[95 \% \mathrm{CI}(\beta)]$} & $p$ value \\
\hline Erythrocytes $\left(10^{6} / \mathrm{mm}^{3}\right)$ & & $\mathbf{0 . 0 0 6}$ & {$[-\mathbf{0 . 0 9 - 0 . 1 0}]$} & $\mathbf{0 . 8 9}$ \\
& Haemoglobin $(\mathrm{g} / \mathrm{dl})$ & -0.20 & {$[-0.43-0.02]$} & 0.08 \\
& Haematocrit $(\%)$ & -0.61 & {$[-1.25-0.02]$} & 0.06 \\
& MCV $\left(\mu \mathrm{m}^{3}\right)$ & -1.50 & {$[-2.80$ to -0.21$]$} & 0.02 \\
& $\mathrm{MCH}(\mathrm{pg})$ & -0.54 & {$[-1.04$ to -0.03$]$} & 0.04 \\
Thrombocytes $\left(10^{3} / \mathrm{mm}^{3}\right)$ & MCHC $(\mathrm{g} / \mathrm{dl})$ & -0.02 & {$[-0.22-0.17]$} & 0.83 \\
Leukocytes $\left(10^{3} / \mathrm{mm}^{3}\right)$ & & $\mathbf{2 8 . 2}$ & {$[\mathbf{1 0 . 1}-\mathbf{4 6 . 4}]$} & $\mathbf{0 . 0 0 2}$ \\
& & $\mathbf{1 . 1 3}$ & {$[\mathbf{1 . 0 5}-\mathbf{1 . 2 0}]$} & $\mathbf{0 . 0 0 0 3}$ \\
& Lymphocytes $\left(10^{3} / \mathrm{mm}^{3}\right)$ & 1.11 & {$[1.04-1.18]$} & 0.001 \\
& Neutrophils $\left(10^{3} / \mathrm{mm}^{3}\right)$ & 1.12 & {$[1.03-1.22]$} & 0.01 \\
& Eosinophils $\left(10^{3} / \mathrm{mm}^{3}\right)$ & 1.64 & {$[1.36-1.98]$} & $<0.001$ \\
& Basophiles $\left(10^{3} / \mathrm{mm}^{3}\right)$ & 1.27 & {$[1.13-1.43]$} & 0.0001 \\
& Monocytes $\left(10^{3} / \mathrm{mm}^{3}\right)$ & - & - & - \\
\hline
\end{tabular}


related to active defense mechanisms directed against adult worms.

With regard to erythrocytes; although the haemoglobin level was similar in both groups, the FBC yielded significantly lower haemoglobin and haematocrit levels among children infected with schistosomiasis, as compared to those non-infected. Similar erythrocyte profiles were recently reported by Afrifa et al. among Ghanaian children with urogenital schistosomiasis compared to those without [10]. In our study, however, when adjusting for history of PZQ intake, STH and Plasmodium spp. infection status, only a trend towards lower haemoglobin and haematocrit levels was observed; while, lower MCV and MCH levels remain significant. Indeed, the haemoglobin levels observed in this population were similar to the levels observed in children without the disease, and were above $11 \mathrm{~g} / \mathrm{dL}$, the threshold set by the WHO to define anaemia for populations living at sea level [30]. The absence of a statistically relationship between anaemia and schistosomiasis might appear surprising, since the main symptom of urogenital schistosomiasis is hematuria occurring by blood spilling during egg excretion. However, longer intervals between blood losses, few or no macrohematuria episodes and haematological recovery between episodes might be explanatory. Plasmodium spp. infection is usually associated with lower hematocrit and haemoglobin concentrations [31], and could explain the lower level of haemoglobin display by children with schistosomiasis. We indeed found an association between both infections. Interpretation of haemoglobin and platelets levels for one of these two diseases should, therefore, consider the possibility of the presence of both. Similar explanations could apply for cases with STH co-infections.

Some factors such as schistosomiasis chronicity and intensity could influence the relationship between schistosomiasis and FBC parameters we reported here and were not take into account in our analysis, particularly due to the study design. However, the large sample size included in this study should be reassuring with regard to the accuracy of our findings. The study was conducted among schoolchildren knowing to be most affected by the disease and our conclusions are limited to this population. However, further investigations should be conducted particularly among women also known to bear a high burden of urogenital schistosomiasis.

In conclusion, in our setting, schistosomiasis is associated with a characteristic FBC profile, indicating the necessity to consider schistosomiasis as a single cause of disease, or a co-morbidity, when interpreting an FBC in schistosomiasisendemic areas.

Acknowledgements We thank the research team involved in this project for their special commitment during this study. We specially thank the school children for their voluntary participation in this study, their parents, teachers, and to the administrative authorities of health and education for allowing us to carry out this study. AAA, MPG are members of the CANTAM (EDCTP-RegNet2015-1045) network; AAA is member of the PANDORA-ID-Net (EDCTP Grant Agreement RIA2016E-1609) network.

Author contributions JCDA, AAA and MPG conceived the paper. JCDA conducted the primary analysis and wrote the first draft of the manuscript. AAA and MPG contributed to the writing of the first draft and further analyses. All authors contributed to, and endorsed the final version of the manuscript.

Funding No specific funding was received for the work reported here.

\section{Compliance with ethical standards}

Conflict of interest None of the authors (JCDA, AAA and MPG) has any commercial or other association to report that might pose a conflict of interest.

Open Access This article is licensed under a Creative Commons Attribution 4.0 International License, which permits use, sharing, adaptation, distribution and reproduction in any medium or format, as long as you give appropriate credit to the original author(s) and the source, provide a link to the Creative Commons licence, and indicate if changes were made. The images or other third party material in this article are included in the article's Creative Commons licence, unless indicated otherwise in a credit line to the material. If material is not included in the article's Creative Commons licence and your intended use is not permitted by statutory regulation or exceeds the permitted use, you will need to obtain permission directly from the copyright holder. To view a copy of this licence, visit http://creativecommons.org/licenses/by/4.0/.

\section{References}

1. World Health Organization. Schistosomiasis. Available at: http:// www.who.int/mediacentre/factsheets/fs115/en/. Accessed 24 June 2020.

2. World Health Organization. Available at: https://www.who.int/ health-topics/schistosomiasis. Accessed 16 July 2020

3. Hotez PJ, Fenwick A. Schistosomiasis in Africa: an emerging tragedy in our new global health decade. PLoS Negl Trop Dis. 2009;3:e485.

4. Mohammed EH, Eltayeb M, Ibrahim H. Haematological and biochemical morbidity of schistosoma haematobium in school children in Sudan. Sultan Qaboos Univ Med J. 2006;6:59-64.

5. Bierman WFW, Wetsteyn JCFM, van Gool T. Presentation and diagnosis of imported schistosomiasis: relevance of eosinophilia, microscopy for ova, and serology. J Travel Med. 2005;12:9-13.

6. Da'dara AA, Skelly PJ. Schistosomes versus platelets. Thromb Res. 2014; 134:1176-81.

7. Stanley RG, Ngaiza JR, Wambayi E, Lewis J, Doenhoff MJ. Platelets as an innate defence mechanism against Schistosoma mansoni infections in mice. Parasite Immunol. 2003;25:467-73.

8. Joseph M, Auriault C, Capron A, Vorng H, Viens P. A new function for platelets: IgE-dependent killing of schistosomes. Nature. 1983;303:810-2.

9. Souza MR, Toledo CF, Borges DR. Thrombocytemia as a predictor of portal hypertension in schistosomiasis. Dig Dis Sci. 2000;45:1964-70.

10. Afrifa J, Gyedu D, Ofori Gyamerah E, Essien-Baidoo S, Mensah-Essilfie I. Haematological profile and intensity of urogenital 
schistosomiasis in ghanaian children. J Environ Public Health. 2017;2017:4248325.

11. Morenikeji O, Quazim J, Omoregie C, et al. A cross-sectional study on urogenital schistosomiasis in children; haematuria and proteinuria as diagnostic indicators in an endemic rural area of Nigeria. Afr Health Sci. 2014;14:390-6.

12. Hotez PJ, Kamath A. Neglected tropical diseases in sub-saharan Africa: review of their prevalence, distribution, and disease burden. PLoS Negl Trop Dis. 2009;3:e412.

13. Dejon-Agobé JC, Honkpehedji YJ, Zinsou JF, et al. Epidemiology of schistosomiasis and soil-transmitted helminth coinfections among school children living in Lambaréné. Gabon Am J Trop Med Hyg. 2020;103:325-33.

14. Ateba Ngoa U, Zinsou JF, Kassa RFK, et al. Assessment of the effect of Schistosoma haematobium co infection on malaria parasites and immune responses in rural populations in Gabon: study protocol. SpringerPlus. 2014;3:388.

15. Ateba-Ngoa U, Jones S, Zinsou JF, et al. Associations between helminth infections, plasmodium falciparum parasite carriage and antibody responses to sexual and asexual stage malarial antigens. Am J Trop Med Hyg. 2016;95:394-400.

16. Mombo-Ngoma G, Honkpehedji J, Basra A, et al. Urogenital schistosomiasis during pregnancy is associated with low birth weight delivery: analysis of a prospective cohort of pregnant women and their offspring in Gabon. Int $\mathrm{J}$ Parasitol. 2017;47:69-74.

17. Adegnika AA, Agnandji ST, Chai SK, et al. Increased prevalence of intestinal helminth infection during pregnancy in a Sub-Saharan African community. Wien Klin Wochenschr. 2007;119:712-6.

18. Zoleko Manego R, Koehne E, Kreidenweiss A, et al. Description of Plasmodium falciparum infections in central Gabon demonstrating high parasite densities among symptomatic adolescents and adults. Malar J. 2019;18:371.

19. Dejon-Agobé JC, Edoa JR, Honkpehedji YJ, et al. Schistosoma haematobium infection morbidity, praziquantel effectiveness and reinfection rate among children and young adults in Gabon. Parasit Vectors. 2019;12:577.

20. Mbong Ngwese M, Prince Manouana G, Nguema Moure PA, Ramharter M, Esen M, Adégnika AA. Diagnostic techniques of soil-transmitted helminths: impact on control measures. Trop Med Infect Dis. 2020;5:93.

21. Joanny F, Löhr SJZ, Engleitner T, Lell B, Mordmüller B. Limit of blank and limit of detection of Plasmodium falciparum thick blood smear microscopy in a routine setting in Central Africa. Malar J. 2014;13:234.

22. Adegnika AA, Kremsner PG. Epidemiology of malaria and helminth interaction: a review from 2001 to 2011. Curr Opin HIV AIDS. 2012;7:221-4.

23. Adegbite BR, Edoa JR, Honkpehedji YJ, et al. Monitoring of efficacy, tolerability and safety of artemether-lumefantrine and artesunate-amodiaquine for the treatment of uncomplicated Plasmodium falciparum malaria in Lambaréné, Gabon: an open-label clinical trial. Malar J. 2019;18:424.

24. Harris PA, Taylor R, Thielke R, Payne J, Gonzalez N, Conde JG. Research electronic data capture (REDCap)-a metadata-driven methodology and workflow process for providing translational research informatics support. J Biomed Inform. 2009;42:377-81.

25. ICH. Official web site. ICH. Available at: https://www.ich.org/ home.html. Accessed 24 Apr 2020.

26. The World Medical Association. Déclaration d'Helsinki de L'AMM: Principes éthiques applicables à la recherche médicale impliquant des êtres humains. Available at: https://www.wma.net/ fr/policies-post/declaration-dhelsinki-de-lamm-principes-ethiq ues-applicables-a-la-recherche-medicale-impliquant-des-etres -humains/. Accessed 23 Apr 2020.

27. Alwajeeh, et al. Uncomplicated falciparum malaria among schoolchildren in Bajil district of Hodeidah governorate, west of Yemen: association with anaemia and underweight. Malar J. 2020;19(1):358. https://doi.org/10.1186/s12936-020-03431-1.

28. Hagan P, Wilkins HA, Blumenthal UJ, Hayes RJ, Greenwood BM. Eosinophilia and resistance to Schistosoma haematobium in man. Parasite Immunol. 1985;7:625-32.

29. Issa RM, Shalaby MA. Eosinophilia as a diagnostic value in patients suffering from schistosomiasis haematobium comparing to eosinophiluria and egg count in the urine. J Egypt Soc Parasitol. 1999;29:431-49.

30. WHO. Haemoglobin concentrations for the diagnosis of anaemia and assessment of severity. Available at: http://www.who.int/ vmnis/indicators/haemoglobin.pdf. Accessed 15 March 2020.

31. Igbeneghu $\mathrm{C}$, Odaibo $\mathrm{AB}$, Olaleye $\mathrm{DO}$. Impact of asymptomatic malaria on some hematological parameters in the Iwo community in Southwestern Nigeria. Med Princ Pract Int J Kuwait Univ Health Sci Cent. 2011;20:459-63. 